\title{
New constructions of homogeneous operators
}

\author{
Adam Korányi ${ }^{a}$ Gadadhar Misra ${ }^{\mathrm{b}}$ \\ ${ }^{\mathrm{a}}$ Lehman College \\ Bronx, NY 10468 \\ ${ }^{\mathrm{b}}$ Indian Statistical Institute \\ R. V. College Post \\ Bangalore 560059
}

\begin{abstract}
New examples of homogeneous operators involving infinitely many parameters are constructed. They are realized on Hilbert spaces of holomorphic functions with reproducing kernels which are computed explicitly. All the examples are irreducible and belong to the Cowen - Douglas class. Even though the construction is completely explicit, it is based on certain facts about Hermitian holomorphic homogeneous vector bundles. These facts also make possible a description of all homogeneous Cowen - Douglas operators, in a somewhat less explicit way.

Ou construit une nouvelle famille d'examples d'opérateurs homogènes dépendant d'une infinité de paramètres. Les exemples sont réalisés sur des espaces de fonctions holomorphes possédant des noyaux reproduisants qu'on calcule explicitement. Les exemples sont tous des opérateurs irréductibles appartenant à la classe de Cowen Douglas. Tout en étant complètement explicite, la construction est fondée sur certaines propriétés des fibrés vectoriels hermitiens holomorphes homogènes. Ces propriétés permettent aussi une description, un peu moins explicite, de tous les opérateurs homogènes de la classe de Cowen - Douglas.
\end{abstract}

An operator $T$ on a Hilbert space is said to be homogeneous if its spectrum is contained in the closure of the unit disc $\mathbb{D}$ in $\mathbb{C}$ and if $g(T)$ is unitarily equivalent to $T$ for every element $g$ of the holomorphic automorphism group $G$ of $\mathbb{D}$. There are general results about such operators, but relatively few examples are known (cf. $[7,2,3,1])$. In this note a large family of examples is constructed and a step is made towards the description of all such operators in the Cowen - Douglas class of $\mathbb{D}$ (see [4]).

\section{Construction of the examples}

Let $\tilde{G}$ denote the universal covering group of $G$. For $\lambda>0$, let $A^{(\lambda)}$ denote the Hilbert space of holomorphic functions on $\mathbb{D}$ with reproducing kernel $(1-z \bar{w})^{-2 \lambda}$. The well-known discrete series $D_{\lambda}^{+}$of unitary representation of $\tilde{G}$ acts on $A^{(\lambda)}$ by

Email addresses: adam.koranyi@lehman.cuny.edu (Adam Korányi), gm@isibang.ac.in (Gadadhar Misra). 
with the power defined to ensure continuity on $\tilde{G} \times \mathbb{D}$.

$$
\left(D_{\lambda}^{+}(g) f\right)(z)=\left(\frac{\partial\left(g^{-1} z\right)}{\partial z}\right)^{\lambda} f\left(g^{-1}(z)\right)
$$

Let $m \geq 1$ be an integer and let $\lambda>\frac{m}{2}$. For $0 \leq j \leq m$ we write $\lambda_{j}=\lambda-\frac{m}{2}+j$ and define the operator $\Gamma_{j}: A^{\left(\lambda_{j}\right)} \rightarrow \operatorname{Hol}\left(\mathbb{D}, \mathbb{C}^{m+1}\right)$ by

$$
\left(\Gamma_{j} f\right)_{\ell}=\left\{\begin{array}{ll}
0 & \text { if } \ell<j \\
\left(\begin{array}{l}
\ell \\
j
\end{array}\right) \frac{1}{\left(2 \lambda_{j}\right)} f_{\ell-j} f^{(\ell-j)} & \text { if } \ell \geq j
\end{array} .\right.
$$

Denote by $\mathbf{A}^{\left(\lambda_{j}\right)}$ the image of $\Gamma_{j}$. The algebraic sum of the spaces $\mathbf{A}^{\left(\lambda_{j}\right)}$ can be shown to be direct. Hence, for any $\mu_{1}, \ldots, \mu_{m}$ and $\mu_{0}=1$ we can define a norm on the direct sum by

$$
\left\langle\sum_{j=0}^{m} \Gamma_{j} f_{j}, \sum_{j=0}^{m} \Gamma_{j} g_{j}\right\rangle=\sum_{j=0}^{m} \mu_{j}^{2}\left\langle f_{j}, g_{j}\right\rangle, f_{j}, g_{j} \in A^{\left(\lambda_{j}\right)} .
$$

We denote the Hilbert space obtained in this way by $\mathbf{A}^{(\lambda, \boldsymbol{\mu})}$ (we write $\boldsymbol{\mu}=\left(\mu_{0}, \mu_{1}, \ldots, \mu_{m}\right)$ ).

The direct sum of maps $\Gamma:=\oplus \mu_{j} \Gamma_{j}$ is then a Hilbert space isomorphism of $\oplus A^{\left(\lambda_{j}\right)}$ onto $\mathbf{A}^{(\lambda, \mu)}$. This isomorphism transfers the representation $\oplus D_{\lambda_{j}}^{+}$to $\mathbf{A}^{(\lambda, \mu)}$; we denote its image by $U$.

For $g \in \tilde{G}$, we have $g^{\prime \prime}(z)=-2 c g^{\prime}(z)^{3 / 2}$ with a constant $c$ depending on $g$. We use $c$ with this meaning in the following theorem.

Theorem $1.1 U$ is a multiplier representation of $\tilde{G}$, that is, $(U(g) f)(z)=J\left(g^{-1}, z\right) f\left(g^{-1}(z)\right)$ for $g \in$ $\tilde{G}, z \in \mathbb{D}$. The matrix entries of the multiplier are given by

$$
J(g, z)_{p, \ell}= \begin{cases}0 & \text { if } p<\ell \\
\left(\begin{array}{l}
p \\
\ell
\end{array}\right)(-c)^{p-\ell} g^{\prime}(z)^{\lambda-\frac{m}{2}+\frac{p+\ell}{2}} & \text { if } p \geq \ell .\end{cases}
$$

The theorem is proved by direct computation.

It is well known that in each $A^{\left(\lambda_{j}\right)}$ the monomials, appropriately normalized, form an orthonormal basis. Using the isomorphism $\Gamma$ we obtain an orthonormal basis of $\mathbf{A}^{(\lambda, \mu)}$.

Explicitly, the basis is $\left\{\mu_{j} e_{n}^{j}\right\}, 0 \leq j \leq m, n \geq 0$, where the $\ell^{\prime}$ th component of $e_{n}^{j}$ is

$$
e_{n}^{j}(z)_{\ell}= \begin{cases}0 & \text { if } \ell<j \text { or } \ell>n+j \\
\left(\begin{array}{l}
\ell \\
j
\end{array}\right) \frac{\sqrt{n !}}{(n-\ell+j) !} \frac{\sqrt{\left(2 \lambda_{j}\right)_{n}}}{\left(2 \lambda_{j}\right)_{\ell-j}} z^{(n-\ell+j)} & \text { if } j \leq \ell \leq n+j .\end{cases}
$$

Here $(x)_{p}:=x(x+1) \cdots(x+p-1)$ is the Pochhammer symbol. It follows that the polynomials form a dense set in $\mathbf{A}^{(\lambda, \boldsymbol{\mu})}$. Therefore, the linear operator $M=M^{(\lambda, \boldsymbol{\mu})}$ on $\mathbf{A}^{(\lambda, \boldsymbol{\mu})}$ defined by

$$
(M f)(z)=z f(z)
$$

is densely defined. To find the expression of $M$ in terms of our orthonormal basis, we define the subspace $H(n)$ as the linear span of the vectors $\left\{e_{n-j}^{j}: 0 \leq j \leq \min (n, m)\right\}$ for each $n \geq 0$. Clearly $M$ maps $H(n)$ to $H(n+1)$. We have

$$
M \mu_{j} e_{n-j}^{j}=\sum_{n=0}^{m} M(n)_{k, j} \mu_{k} e_{n+1-k}^{k} .
$$

We define the number $E(n)_{\ell j}$ by $\left(e_{n-j}^{j}(z)\right)_{\ell}=E(n)_{\ell j} z^{n-\ell}$ and write $E(n)$ for the matrix $E(n)_{\ell j}$. We define the diagonal matrix $D(\mu)$ by $D(\mu)_{\ell j}=\mu_{\ell} \delta_{\ell j}$. Now we have for $n \geq m$

$$
M(n)=D(\mu)^{-1} E(n+1)^{-1} E(n) D(\mu),
$$


(with a small modification for $0 \leq n<m$ ). Using Stirling's formula one verifies that, as $n \rightarrow \infty$,

$$
M(n) \sim I_{m+1}+O\left(\frac{1}{n}\right),
$$

where $O\left(\frac{1}{n}\right)$ stands for an $(m+1) \times(m+1)$ matrix whose entries are $O\left(\frac{1}{n}\right)$.

Theorem 1.2 The operator $M=M^{(\lambda, \mu)}$ defined on $\mathbf{A}^{(\lambda, \mu)}$ by $(M f)(z)=z f(z)$ belongs to the Cowen Douglas class and is homogeneous.

For the proof one remarks that by (1), the operator $M$ splits into the sum of two operators. The first one is the direct sum of $(m+1)$ copies of the standard isometric shift operator and the second one belongs to the Hilbert-Schmidt class. This implies that it is bounded and is in the Cowen - Douglas class.

Using Theorem 1.1 one can verify, in a standard way, that $g(M)=U_{g}^{-1} M U_{g}$ proving that $M$ is homogeneous.

\section{Inequivalence}

Theorem 2.1 For every $m \geq 1$, the operators $M^{(\lambda, \boldsymbol{\mu})}, \lambda>\frac{m}{2} ; \mu_{1}, \ldots, \mu_{m}>0$ are mutually unitarily inequivalent.

One essential ingredient of the proof is the following.

Theorem 2.2 Suppose $E$ is a Hermitian holomorphic vector bundle over $\mathbb{D}$ and for every $g \in G$ there exists an automorphism $\hat{g}$ of $E$ whose action on $\mathbb{D}$ coincides with $g$. Then $\tilde{G}$ acts on $E$ by automorphisms in a unique way.

In the proof one considers the connected component $\hat{G}$ of the full automorphism group of $E$. There is a natural homomorphism $\hat{G} \rightarrow G$ which has a compact kernel $N$. One shows that $\hat{G}$ is reductive so it contains a normal subgroup which is a covering of $G$.

For the proof of Theorem 2.1 we consider the representation of the Lie algebra $\mathfrak{g}$ induced by $U$ and extend it to the complexification $\mathfrak{g}^{\mathbb{C}}=\mathfrak{s l}(2, \mathbb{C})$, still denoting it by $U$.

Restricting $U$ to the triangular subalgebra $\mathfrak{t}$ of $\mathfrak{g}^{\mathbb{C}}$ (the Lie algebra of the stabilizer of 0 in $S L(2, \mathbb{C}$ ) acting on the extended complex plane), direct computation using Theorem 1.1 shows that $(U(X) f)(0)=$ $\rho_{\lambda, \mu}(X) f(0)$ for all $X \in \mathfrak{t}$ and $f \in \mathbf{A}^{(\lambda, \mu)}$, with $\rho_{\lambda, \mu}(X)$ independent of $f$, and therefore, giving a representation of $\mathfrak{t}$ on the finite dimensional Hilbert space $\mathbb{C}^{m+1}$. The unitary equivalence class of $\rho_{\lambda, \boldsymbol{\mu}}$ is uniquely determined by $U$, and hence by the operator $M^{(\lambda, \mu)}$.

Explicit computation of $\rho_{\lambda, \mu}$ shows that different pairs $(\lambda, \boldsymbol{\mu})$ give inequivalent representations, proving the theorem.

\section{Reproducing kernel and irreducibility}

In the following theorem $S$ stands for the matrix with entries $S_{\ell p}=\delta_{p+1, \ell} \ell, T$ its transpose. $D=D(z \bar{w})$ is diagonal with entries $D_{\ell \ell}=(1-z \bar{w})^{m-\ell}$. Let $K^{\lambda, \mu}$ be the diagonal matrix with

$$
\left(K^{\lambda, \mu}\right)_{\ell \ell}=\sum_{j=0}^{m} \mu_{j}^{2} \sum_{j=0}^{\ell}\left(\begin{array}{l}
\ell \\
j
\end{array}\right)^{2} \frac{(\ell-j) !}{\left(2 \lambda_{j}\right)_{\ell-j}} .
$$

Theorem 3.1 The space $\mathbf{A}^{(\lambda, \boldsymbol{\mu})}$ has a reproducing kernel given by

$$
\mathbf{B}^{(\lambda, \boldsymbol{\mu})}(z, w)=(1-z \bar{w})^{-2 \lambda-m} D(z \bar{w}) e^{\bar{w} S} K^{\lambda, \mu} e^{z T} D(z \bar{w}) .
$$

The normalized reproducing kernel in the sense of [5] is 


$$
\mathbf{B}_{0}^{(\lambda, \boldsymbol{\mu})}(z, w)=e^{-z T}\left(K^{\lambda, \boldsymbol{\mu}}\right)^{-1} \mathbf{B}^{(\lambda, \boldsymbol{\mu})}(z, w)\left(K^{\lambda, \boldsymbol{\mu}}\right)^{-1} e^{-\bar{w} S} .
$$

This is proved by computing the effect of the map $\Gamma$ on the well known reproducing kernels of the spaces $A^{\left(\lambda_{j}\right)}$ and using the identity

$$
\mathbf{B}^{(\lambda, \boldsymbol{\mu})}(z, w)=J(g, z) \mathbf{B}^{(\lambda, \boldsymbol{\mu})}(g z, g w) J(g, w)^{*}, g \in \tilde{G} .
$$

Theorem 3.2 The operators $M^{(\lambda, \boldsymbol{\mu})}, \lambda>\frac{m}{2} ; \mu_{1}, \ldots, \mu_{m}>0$ are irreducible.

One proves this by first refining some arguments of [5] to show that any orthogonal projection commuting with $M$ corresponds to an orthogonal projection $P$ in $\mathbb{C}^{m+1}$ such that $P \mathbf{B}_{0}^{(\lambda, \boldsymbol{\mu})}(z, w)=\mathbf{B}_{0}^{(\lambda, \boldsymbol{\mu})}(z, w) P$ for all $z, w \in \mathbb{D}$. Such a $P$ then commutes with all coefficients of the power series development $\mathbf{B}_{0}^{(\lambda, \boldsymbol{\mu})}$ at $z=w=0$. Using the explicit expressions in Theorem 5 one can show that no non-trivial $P$ can exist.

\section{More general results}

Every Hermitian holomorphic vector bundle homogeneous under $\tilde{G}$ can be obtained by the process of holomorphic induction [6, Ch. 13] from representations of $\mathfrak{t}$ on $\mathbb{C}^{m+1}$ that are Hermitian on the real reductive part of $\mathfrak{t}$. Unitary equivalence classes of such representations are in one-to-one correspondence with isomorphism classes of bundles. The $\rho_{\lambda, \boldsymbol{\mu}}$ occurring above in Section 2 are certain conjugates of the restriction of the standard $(m+1)$-dimensional irreducible representation of $\mathfrak{s l}(2, \mathbb{C})$ to $\mathfrak{t}$ tensored with a one-dimensional representation. Any finite dimensional representation of $\mathfrak{t}$ can be written as a tensor product of a one-dimensional representation $\rho_{\lambda}$ characterized by a real parameter $\lambda$ and another one $\rho_{0}$ normalized in some way.

Even though the results are less explicit in the general case than for the operators constructed in Sections 1 and 2, we can prove the following.

Theorem 4.1 To any $\rho_{0}$ there corresponds a number $\lambda_{0}$ such that the homogeneous bundle induced by $\rho_{\lambda} \otimes \rho_{0}$ for $\lambda>\lambda_{0}$ is the Cowen - Douglas bundle of a homogeneous operator.

It is also possible to make statements about irreducibility and inequivalence of such operators.

A number of results in this Note extend to the case of operator tuples of Cowen - Douglas class on bounded symmetric domains in several complex variables.

Details will appear elsewhere.

\section{References}

[1] B. Bagchi and G. Misra, Homogeneous tuples of multiplication operators on twisted Bergman space, J. Funct. Anal. 136 (1996), $171-213$.

[2] B. Bagchi and G. Misra, Homogeneous operators and projective representations of the Möbius group: a survey, Proc. Ind. Acad. Sc.(Math. Sci.) 111 (2001), 415-437.

[3] _ The homogeneous shifts, J. Funct. Anal. 204 (2003), 293 -319.

[4] M. J. Cowen and R. G. Douglas, Complex geometry and operator theory, Acta Math. 141 (1978), 187-261.

[5] R. E. Curto and N. Salinas, Generalized Bergman kernels and the Cowen - Douglas theory, Amer J. Math. 106 (1984), 447-488.

[6] A. A. Kirillov, Elements of the theory of representations, Grundlehren der Mathematischen Wissenschaften, Band 220. Springer-Verlag, Berlin-New York, 1976.

[7] D. R. Wilkins, Homogeneous vector bundles and Cowen - Douglas operators, International J. of Math. 4 (1993), 503 520 . 